\title{
Zerumbone suppresses the potential of growth and metastasis in hepatoma HepG2 cells via the MAPK signaling pathway
}

\author{
TAO LV* ${ }^{*}$, WENJING ZHANG ${ }^{*}$ and XIAOLI HAN \\ Department of General Surgery, Yantai Yeda Hospital, Yantai, Shandong 264006, P.R. China
}

Received July 25, 2016; Accepted January 11, 2018

DOI: $10.3892 / \mathrm{ol} .2018 .8335$

\begin{abstract}
Zerumbone is an active component of Zingiber zerumbet (L.) Smith and can perform a diverse range of antitumor activities. However, the underlying molecular mechanisms of zerumbone action have not yet been elucidated. The aim of the present study was to investigate the antitumor effects, and the associated molecular mechanisms, of zerumbone in hepatoma HepG2 cells. Treatment with zerumbone markedly induced apoptosis in hepatoma HepG2 cells and suppressed their invasion and metastasis in a dose-dependent manner. Further investigation revealed that treatment with zerumbone led to the dose-dependent induction of apoptosis and cell cycle arrest at $\mathrm{G}_{2} / \mathrm{M}$ phase in cancer cells. Zerumbone treatment led to the increased expression of p27, cytochrome c, caspase-3 and-9, and Bcl-2-associated X expression, but the decreased expression of cyclin-dependent kinase 1 , cyclin B1, B-cell lymphoma-2, focal adhesion kinase, Ras homolog gene family, member A, Rho-associated protein kinase-1, and matrix metalloproteinase- 2 and-9 in HepG 2 cells. In addition, the phosphorylation of p38 mitogen-activated protein kinase and extracellular signal-regulated kinase $1 / 2$, but not C-Jun N-terminal kinase $1 / 2$, was regulated in a dose-dependent manner in response to zerumbone treatment. The results of the current study indicate that zerumbone could be used as potential anticancer agent in for the treatment of hepatoma in the future.
\end{abstract}

\section{Introduction}

Hepatoma is a malignant tumor that occurs more frequently in children than adults; it often cannot be completely excised and the 5-year survival rate was $\sim 40 \%$ in China (1). Multiple factors are involved in the carcinogenesis of hepatoma, including genetic susceptibility and environmental factors $(2,3)$.

Correspondence to: Professor Xiaoli Han, Department of General Surgery, Yantai Yeda Hospital, 23-1 Huanghe, Yantai, Shandong 264006, P.R. China

E-mail: hedlcl@163.com

*Contributed equally

Key words: zerumbone, hepatoma, tumor metastasis
Currently, although multiple therapeutic methods, including surgery and chemotherapy, are available for the treatment of hepatoma, the prognosis is often not satisfactory owing to the rates of recurrence and metastasis $(4,5)$. Thus, the development of novel drugs with high efficacy and low toxicity for the treatment of hepatoma is required.

Traditional Chinese medicines (TCMs) have been used to treat cancer in China for thousands of years owing to their high efficacy and low toxicity $(6,7)$. Certain natural products have been widely used for the treatment of cancer, including hepatoma $(8,9)$. Zerumbone, a monocyclic sesquiterpene derived from Zingiber zerumbet (L.) Smith, is reported to produce a variety of pharmacological effects, including antioxidant, antiviral, anti-inflammatory antibacterial activities (10). Notably, zerumbone has been reported to have anticancer efficacy in certain cancer cell lines, including those of breast, bladder and brain cancer $(11,12)$; however, there are limitations to the antitumor mechanism.

The present study aimed to investigate the effect of zerumbone on the proliferation, cell cycle distribution and apoptosis of hepatoma HepG2 cells, and assess its possible mechanism in vitro. These data demonstrated that zerumbone could inhibit HepG2 cell proliferation and metastasis of hepatoma by inducing apoptosis. Furthermore, it was also demonstrate that zerumbone induced apoptosis through the mitogen-activated protein kinase (MAPK)-extracellular signal-regulated kinase (ERK) pathways. In conclusion, the data of the present study indicated that zerumbone may be a useful plant product for the treatment of hepatoma.

\section{Materials and methods}

Cell culture. The human hepatoma HepG2 cell line was purchased from the Type Culture Collection of the Chinese Academy of Sciences (Shanghai, China). Cells were maintained in Dulbecco's Modified Eagle's Medium (DMEM; Gibco; Thermo Fisher Scientific, Inc., Waltham, MA, USA) supplemented with $10 \%$ fetal bovine serum (FBS; Gibco; Thermo Fisher Scientific, Inc.) in a humidified incubator under $5 \% \mathrm{CO}_{2}$ at $37^{\circ} \mathrm{C}$.

MTT assay for cell viability. The cytotoxicity of zerumbone was detected by MTT assay. Briefly, HepG2 cells were seeded in 96-well culture plates at a density of $4 \times 10^{4}$ cells per well at $37^{\circ} \mathrm{C}$ for $24 \mathrm{~h}$. Next, cells were incubated with different concentrations 
$(0,2,5,10,20,50,100 \mu \mathrm{M})$ of zerumbone in culture medium for $48 \mathrm{~h}$. Following this, $20 \mu \mathrm{l} /$ well of MTT solution (dissolved in PBS) was added and the plates were incubated at $37^{\circ} \mathrm{C}$ for $4 \mathrm{~h}$. The absorbance was measured at $490 \mathrm{~nm}$ with a microplate reader to assess the optical density (OD), from which cell counts were determined. The inhibition rate of zerumbone on these cells was calculated as follows: Inhibition rate $(\%)=(1$-experimental group OD/control group OD) x100.

Flow cytometry analysis. Cells were treated with $0.1 \%$ DMSO control or zerumbone (10 or $30 \mu \mathrm{M})$ for $48 \mathrm{~h}$, and then fixed overnight with $70 \%$ ethanol in ice-cold PBS at $-20^{\circ} \mathrm{C}$, and then resuspended with $50 \mu \mathrm{g} / \mathrm{ml}$ of propidium iodide (PI) (Sigma-Alrich; Merck KgaA, Darmstadt, Germany) and $50 \mu \mathrm{g} / \mathrm{ml}$ of RNAse A (Sangon Biotech Co., Ltd., Shanghai, China) and incubated in the dark for $15 \mathrm{~min}$ at room temperature. The DNA contents of samples were analyzed using a FACSCalibur flow cytometer (BD Biosciences, San Jose, CA, USA). Apoptosis was determined using Annexin V-FITC. Following treatment of HepG2 cells with different concentrations $(10$ or $30 \mu \mathrm{M})$ of zerumbone for $48 \mathrm{~h}$. Cells were washed twice with PBS at $4^{\circ} \mathrm{C}$ and the supernatant was discarded. Add $100 \mu \mathrm{l}$ pre-cooled binding buffer (EMD Millipore, Billerica, MA, USA) to re-suspend the cells for ice bath. Next, $10 \mu \mathrm{l}$ Annexin V-fluorescein isothiocyanate (FITC; EMD Millipore) and $10 \mu 1$ PI were added to the cell suspension and gently mixed. The calls were then incubated at room temperature for 15 min dark staining, after which another $400 \mu \mathrm{l}$ binding buffer was added to re-suspend the cells. Next, Multimode Plate Reader (Varioskan LUX; Thermo Fisher Scientific Inc., Waltham, MA, USA) was used to detect the percentage of apoptotic cells. The experiment was repeated three times.

Cell adhesion assay. A cell adhesion assay were performed, as previously described (3). Briefly, each well of a 96-well plate was coated with $10 \mu l$ fibronectin (R\&D Systems, Inc., Minneapolis, MN, USA), and the plates were incubated at $37^{\circ} \mathrm{C}$ for 2 days and washed twice with DMEM. HepG2 cells were pretreated with 0,10 or $30 \mu \mathrm{M}$ zerumbone for $48 \mathrm{~h}$ at $37^{\circ} \mathrm{C}$, cells were harvested and then seeded into the 96-well fibreonectin-coated plate at a density of $5 \times 10^{5}$ cells $/ \mathrm{ml}$ for $100 \mu \mathrm{l}$. After $2 \mathrm{~h}$, the plates were washed and $10 \mu \mathrm{l}$ MTT $(5 \mathrm{mg} / \mathrm{ml}$ ) solution (dissolved in PBS) was added to the adhered cells. Absorbance was detected at $570 \mathrm{~nm}$ using the Multilabel counter after a 4-h incubation. Values reported are from 3 independent experiments.

Cell migration assay. HepG2 cells were pretreated with 0,10 or $30 \mu \mathrm{M}$ zerumbone for $48 \mathrm{~h}$ at $37^{\circ} \mathrm{C}$. Cells were collected and adjusted to a single-cell suspension of $5 \times 10^{5} / \mathrm{ml}$ cells. Next, $100-\mu l$ cell suspension was placed in each insert upper chamber without matrigel (Corning Incorporated, Corning, NY, USA) containing $200 \mu \mathrm{l}$ FBS free DMEM, whereas $600 \mu \mathrm{l}$ DMEM in the lower chamber contained $10 \%$ FBS. After incubating for $6 \mathrm{~h}$, the chamber was washed twice with PBS, and the cells on the apical side of each insert were scraped off using cotton buds. The cells that had migrated and attached to the lower surface of the insert were fixed with $4 \%$ formaldehyde $10 \mathrm{~min}$ and stained with $0.1 \%$ crystal violet for $20 \mathrm{~min}$ at room temperature. After washing with PBS and air-drying, the number of cells that had migrated through the membrane was counted randomly in five fields under a light microscope (magnification, $\mathrm{x} 400$ ). The migration rate was calculated using the following formula: Migration rate $(\%)=$ number of migrated cell/number of inoculated cells x100.

Cell invasion assay. For the cell invasion assay, transwell filters (Corning Incorporated) were coated with $100 \mu 1$ Matrigel $(300 \mu \mathrm{g} / \mathrm{ml})$ on the upper surface of a polycarbonic membrane. The chambers were placed into a $37^{\circ} \mathrm{C}$ incubator for $2 \mathrm{~h}$ prior to use. Next, $5 \times 10^{4}$ cells in $200 \mu 1$ low serum (1\% FBS) DMEM containing $0.1 \%$ DMSO (control), 10 or $30 \mu \mathrm{M}$ zerumbone was added to the upper chamber, and $600 \mu 1$ medium containing $10 \% \mathrm{FBS}$ was added to the lower chambers. Cells were cultured at $37^{\circ} \mathrm{C}$ in a humidified incubator with $5 \% \mathrm{CO}_{2}$ for $24 \mathrm{~h}$. After incubation, invaded cells on the lower side of the membrane were fixed with $4 \%$ paraformaldehyde for $20 \mathrm{~min}$ at room temperature, and then washed with PBS three times before being stained with $0.1 \%$ crystal violet for another $10 \mathrm{~min}$. The stained cells were visualized under an inverted microscope (magnification, x200) and counted in five random fields. The results were averaged for three independent experiments.

Western blot analysis. HepG2 cells were harvested, rinsed twice with ice-cold PBS and re-suspended in radioimmunoprecipitation assay lysis buffer (Sigma-Aldrich; Merck $\mathrm{KGaA}$ ) and $50 \mathrm{ng}$ protein samples were quantified using a bicinchoninic acid assay Sigma-Aldrick; Merck KGaA) and separated by $12 \%$ SDS-PAGE and transferred onto polyvinylidene difluoride membranes. The membranes were dried, pre-blocked with 5\% non-fat milk in PBS-Tween $(0.1 \%)$ overnight at room temperature, then incubated with the following specific antibodies overnight at $4^{\circ} \mathrm{C}$ : Cyclin dependent kinase inhibitor 1B (CDKN1B, also known as p27) $(1: 2,000)$, cyclin-dependent kinase 1 (CDK1, also known as CDC2) $(1: 2,000)$, cyclin B1 $(1: 2,000)$, B-cell lymphoma-2 (Bcl-2) $(1: 2,000)$, Bcl-2-associated X (Bax) $(1: 2,000)$, cytochrome c $(1: 2,000)$, cleaved caspase-3 $(1: 1,000)$, cleaved caspase-9 $(1: 1,000)$, focal adhesion kinase (FAK) $(1: 2,000)$, Ras homolog gene family, member A (RhoA) $(1: 2,000)$, Rho-associated protein kinase-1 (ROCK-1) $(1: 2,000)$, matrix metalloproteinase-2 (MMP-2) (1:2,000), MMP-9 (1:2,000), phosphorylated (p)-p38 MAPK $(1: 1,000)$, p38 MAPK $(1: 1,000)$, p-ERK1/2 (1:1,000), ERK1/2 (1:1,000), p-c-Jun N-terminal kinase (JNK) $(1: 1,000)$, JNK $(1: 1,000)$ and $\beta$-actin $(1: 5,000)$ at $4^{\circ} \mathrm{C}$ overnight followed by incubated with goat anti-mouse horseradish peroxidase (HRP)-conjugated secondary antibody (1:2,000; cat no. ab7068; Abcam, Cambridge, UK) at room temperature for $1 \mathrm{~h}$. Immune complexes were visualized using the Pierce ECL Western Blotting kit (Thermo Fisher Scientific, Inc., Waltham, MA, USA).

Reverse transcription-quantitative polymerase chain reaction (RT-qPCR) analysis. The expression levels of Bcl-2, Bax, cytochrome c, FAK, RhoA, ROCK-1, MMP-2 and MMP-9 were detected by RT-qPCR using ABI Prism 7900 sequence detection system (Applied Biosystems; Thermo Fisher Scientific, Inc.). Total RNA was extracted from the HepG2 cells which were treated by 10 or $30 \mu \mathrm{M}$ zerumbone $48 \mathrm{~h}$ using TRIzol reagent (Invitrogen; Thermo Fisher Scientific, Inc.) according 

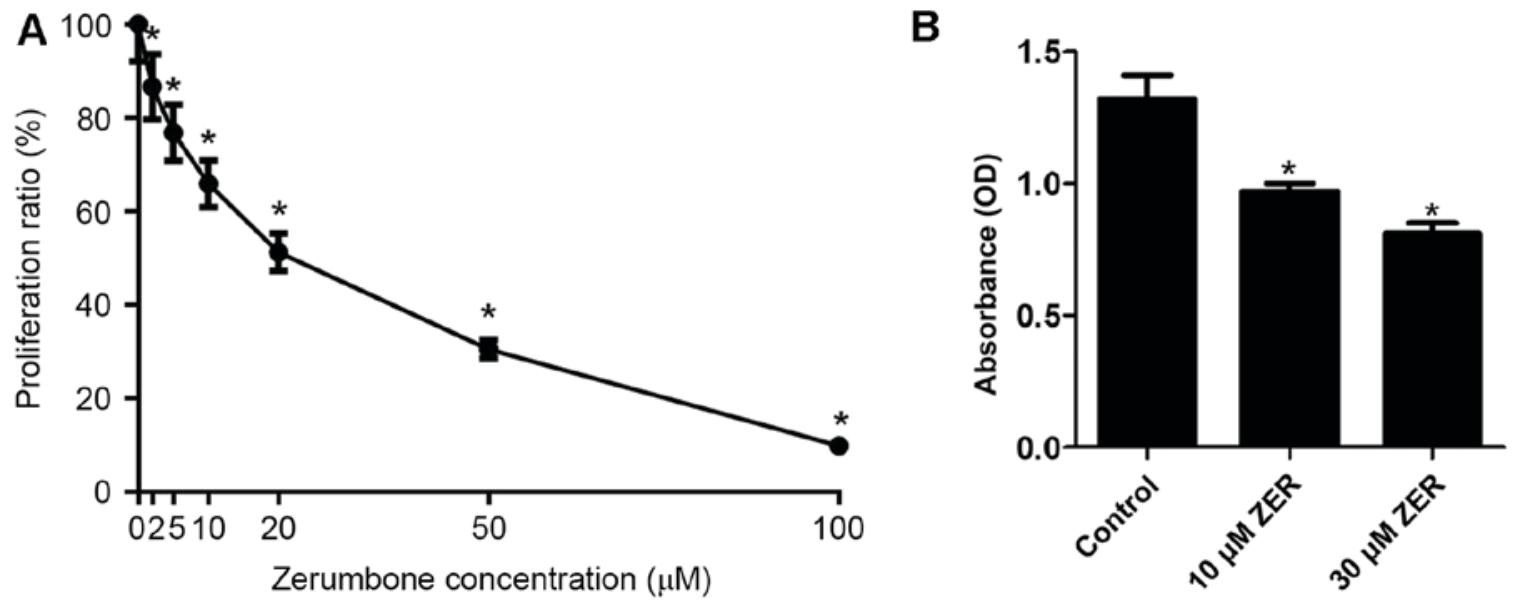

Figure 1. Zerumbone inhibits cell growth in HepG2 cells. Absorbance of the HepG2 cells using a MTT assay for (A) proliferation and (B) adhesion. "P<0.05 vs. control. Data are presented as the mean \pm standard error of the mean.

to the manufacturer's protocol. Subsequently, cDNA was synthesized using M-MLV First Strand cDNA Synthesis kit (Invitrogen; Thermo Fisher Scientific, Inc.). qPCR reactions were performed using a SYBR Premix Ex Taq (Takara Biotechnology Co., Ltd., Dalian, China) according to the manufacturer's protocol. Thermocycling conditions were as follows: Denaturation for $30 \mathrm{sec}$ at $95^{\circ} \mathrm{C}, 20 \mathrm{sec}$ at $95^{\circ} \mathrm{C}, 45 \mathrm{sec}$ at $68^{\circ} \mathrm{C}$ for 30 cycles, $5 \mathrm{~min}$ at $68^{\circ} \mathrm{C}$ for final extension, then $4^{\circ} \mathrm{C}$ for holding. Specific primers for each gene were designed as follows: The PCR primer sets were following: p27 forward, 5'-CTGCCCTCCCCAGTCTCTCT-3' and reverse, 5'-CAA GCACCTCGGATTTT-3'; CDC2 forward, 5'-GCGGCGGGG TACCCCCTGCAGTAAGTGCAGAAATCT-3' and reverse, 5'-CGCCGGAGGATCTTCGCAGCGGCAGCTACAACA AC-3'; cyclin B1 forward, 5'-GCAAATGACAAAGCAAAT GGGG-3' and reverse, 5'-ACAACCAGCAGAAACCAA CAGC-3'; Bcl-2 forward, 5'-CGTACAGTTCCACAAAGG CA-3' and reverse, 5'-ATGTGTGTGGAGAGCGTCAA-3'; Bax forward, 5'-CCCGAGAGGTCTTTTTCCGAG-3' and reverse, 5'-CCAGCCCATGATGGTTCTGAT-3'; cytochrome c forward, 5'-CTTTGGGCGGAAGACAGGTC-3' and reverse 5'-TTATTGGCGGCTGTGTAAGAG-3'; FAK forward, 5'-GCAATTTCCTGGTCCACTTG-3' and reverse, 5'-CGT TATTTGCCAAAAGGATTTC-3'; RhoA forward, 5'-GGA AAGCAGGTAGAGTTGGCT-3' and reverse, 5'-GGCTGT CGATGGAAAAACACAT-3'; ROCK-1 forward, 5'-GGC AGGAAAATCCAAATCAT-3' and reverse, 5'-GGGGAC AGTTTTGAGACTCG-3'; MMP2 forward, 5'-AAGAAGTAG CTGTGACCGCC-3' and reverse, 5'-TTGCTGGAGACAAAT TCTGG-3'; MMP9 forward, 5'-GCACTGCAGGATGTCATA GG-3' and reverse, 5'-ACGACGTCTTCCAGTACCGA-3'; and GAPDH forward, 5'-CCACATCGCTCAGACACCAT-3' and reverse, 5'-ACCAGGCGCCCAATACG-3'. Relative mRNA expression was calculated using the $2^{-\Delta \Delta \mathrm{Cq}}$ method (13), using GAPDH as an endogenous control and untreated samples as the calibrator.

Statistical analysis. The experimental data were analyzed using SPSS 11.0 (SPSS, Inc., Chicago, IL, USA). Data are expressed and the mean \pm standard deviation. The quantitative ratios of different groups were compared using one-way analysis of variance with tukey's post test. $\mathrm{P}<0.05$ was considered to indicate a statistically significant difference. All statistical tests were two sided.

\section{Results}

Zerumbone inhibits the proliferation of HepG2 cells. After cells were treated with different concentrations $(0,2,5,10$, 20,50 or $100 \mu \mathrm{M}, \mathrm{n}=5$ ) of zerumbone for $48 \mathrm{~h}$, the cell growth inhibition for the HepG2 cells is presented in Fig. 1. The results of the MTT assay demonstrated that zerumbone inhibited the growth of HepG2 cells in a concentration-dependent manner (Fig. 1). The half-maximal inhibitory concentration $\left(\mathrm{IC}_{50}\right)$ value for zerumbone was $23.64 \pm 1.23 \mu \mathrm{M}$. Thus, doses higher and lower than the $\mathrm{IC}_{50}(10$ and $30 \mu \mathrm{M})$ were used in subsequent experiments.

Zerumbone induces apoptosis in HepG2 cells. HepG2 cells were cultured in 10 or $30 \mu \mathrm{M}$ zerumbone for $48 \mathrm{~h}$ and the proportion of apoptotic HepG2 cells was then determined using annexin V-FITC staining and flow cytometry. Following treatment of HepG2 cells with 10 or $30 \mu \mathrm{M}$ zerumbone for $48 \mathrm{~h}$, the percentage of apoptotic cells increased from 3.00 to 28.28 or $52.30 \%$ following 10 or $30 \mu \mathrm{M}$ zerumbone treatment, respectively (Fig. 2A). These results indicated that zerumbone treatment could induce apoptosis in HepG2 cells in a concentration-dependent manner.

Zerumbone induced cell cycle arrest at $G_{2} / M$ phase. Cell cycle analysis by flow cytometry showed treatment with 10 or $30 \mu \mathrm{M}$ zerumbone for $48 \mathrm{~h}$ increased the proportion of cells in $\mathrm{G}_{2} / \mathrm{M}$ phase (from $10.05 \pm 0.13$ to $23.1 \pm 0.21$ or $34.96 \% \pm 0.25$ following 10 or $30 \mu \mathrm{M}$ zerumbone treatment, respectively) (Fig. $2 \mathrm{~B}$ and $\mathrm{C}$ ). These results indicated that zerumbone induced cell cycle arrest at $\mathrm{G}_{2} / \mathrm{M}$ in HepG2 cells in a concentration-dependent manner.

Zerumbone reduces the adherence abilities of HepG2 cells. To test the effect of zerumbone on the capacity of cancer cells to bind to the extracellular matrix, a cell adhesion assay was performed in HepG2 cells. As shown in Fig. 2, the adherence 
A

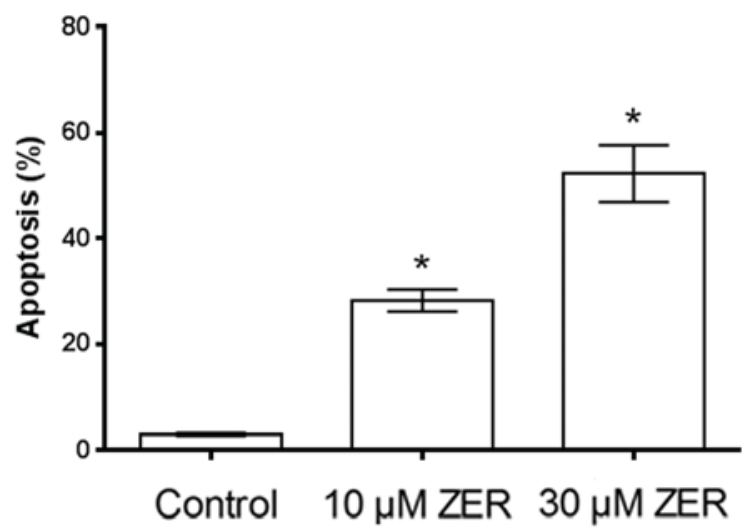

c

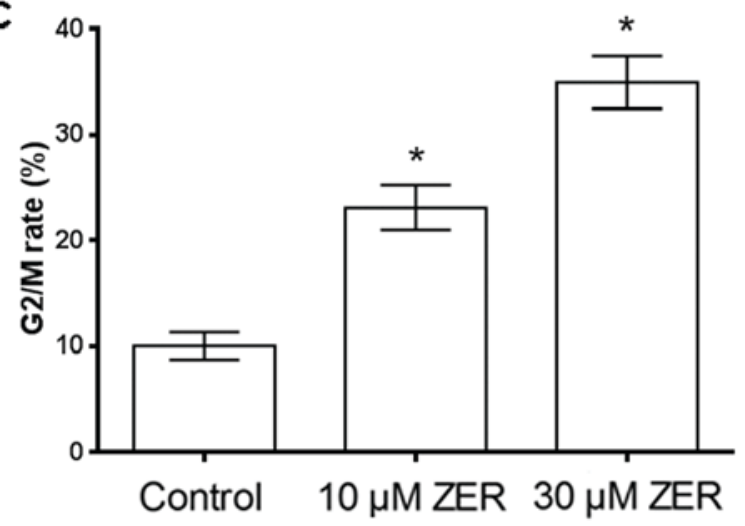

B

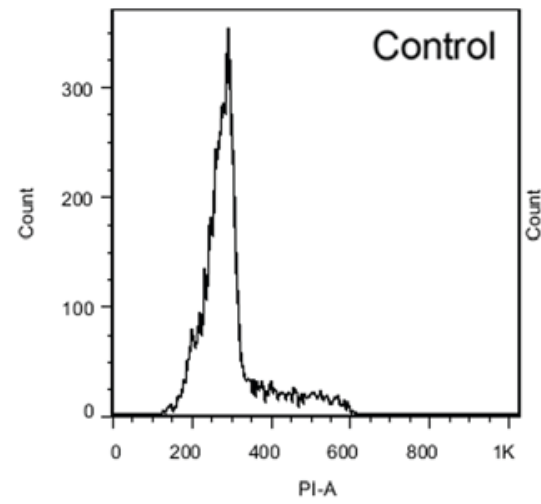

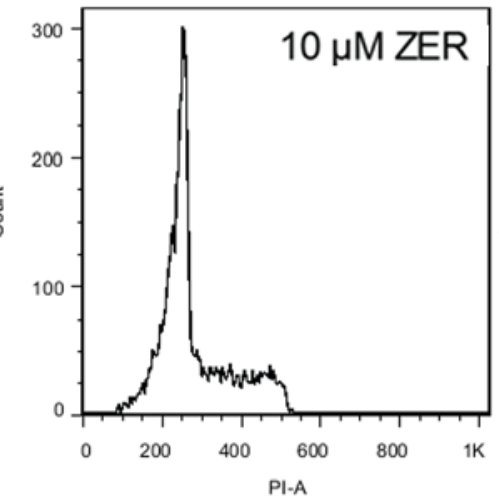

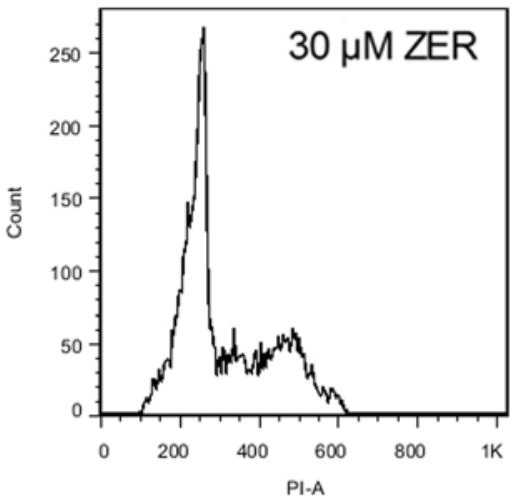

Figure 2. Flow cytometry analysis of HepG2 cells treated with ZER. (A) Histogram of cell apoptosis after treatment with ZER for 48 h by FITC fluorescence. (B) HepG2 cells were incubated with indicated concentrations of ZER for $48 \mathrm{~h}$, stained with PI and analyzed for DNA content by flow cytometry. (C) Cell cycle distribution was monitored by flow cytometry using a PI staining assay. ${ }^{*} \mathrm{P}<0.05$ vs. control. ZER, zerumbone.

abilities of HepG2 cells were significantly reduced following pre-treatment with zerumbone. These results indicated that zerumbone effectively inhibited the adherence ability of HepG2 cells.

Zerumbone inhibits cancer cell migration. Cellular migration was analyzed using a transwell migration assay. As shown in Fig. 3A, compared with the control group, the migratory ability of HepG2 cells was significantly inhibited by zerumbone treatment in a dose-dependent manner $(\mathrm{P}<0.05)$. The results indicated that zerumbone treatment significantly inhibited the migration of the HepG2 cells.

Zerumbone inhibits cancer cell invasion. The effect of zerumbone on cancer cell invasion by was investigated using a transwell invasion assay. As shown in Fig. 3B, the cell invasion rate in the zerumbone-treated groups was significantly lower than those in the control group.

Zerumbone induces activation of caspase-3 and-9 and apoptosis and cell cycle-associated genes in HepG2 cells. The caspase cascade triggered by caspase family members, among them the frequently activated death proteases caspase- 3 and-9, serves a key role in apoptosis (14). To investigate whether caspase activation was involved in zerumbone-induced apoptosis, activation of caspase- 3 and- 9 was detected by western blotting. As shown in Fig. 4A, HepG2 cells treated with zerumbone markedly increased the expression level of cleaved caspase-3 and-9, compared with the control cells. Other apoptosis-and cell cycle-associated genes in HepG2 cells were also investigated by western blotting and RT-qPCR. As shown in Fig. 4, compared to the control, zerumbone treatment significantly increased the expression level of p27, Bax, and cytochrome-c, but decreased the expression level of CDC2, cyclinB1 and Bcl-2. These results indicated that zerumbone induced the activation of caspase-3, caspase-9, cytochrome-c and Bax, and decreased the expression level of Bcl-2, eventually leading to the apoptosis in HepG2 cells.

Zerumbone treatment downregulates the expression levels of FAK, RhoA, ROCK-1,MMP-2 and MMP-9 in Hep G 2 cells. FAK, RhoA, ROCK-1, MMP-2 and MMP-9 are metastasis-associated proteins. The present study determined the effect of zerumbone on the expression of FAK, RhoA, ROCK-1, MMP-2 and MMP-9 by western blotting and RT-qPCR. As depicted in Fig. 5, treatment with zerumbone reduced the mRNA and protein expression of FAK, RhoA, ROCK-1, MMP-2 and MMP-9 in HepG2 cells, compared with the control group.

Zerumbone regulates MAPK and ERK phosphorylation in HepG2 cells. The MAPK signaling pathway serves a notable role in the action of chemotherapeutic drugs. Zerumbone inactivated ERK and activated p38 MAPK in a time-dependent manner (Fig. 6). No effect of zerumbone 
A

Migration

nvasion

B

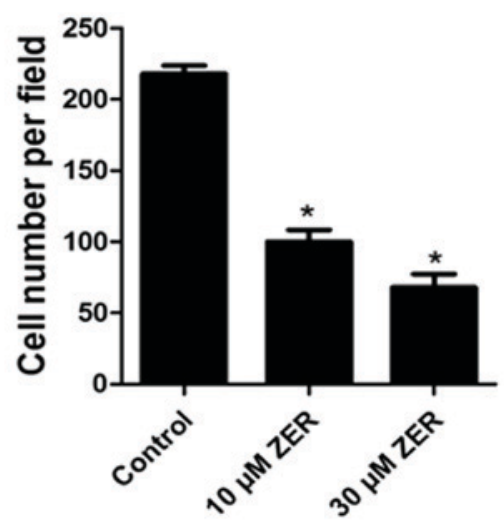

$10 \mu \mathrm{M}$ ZER $\quad 30 \mu \mathrm{M}$ ZER

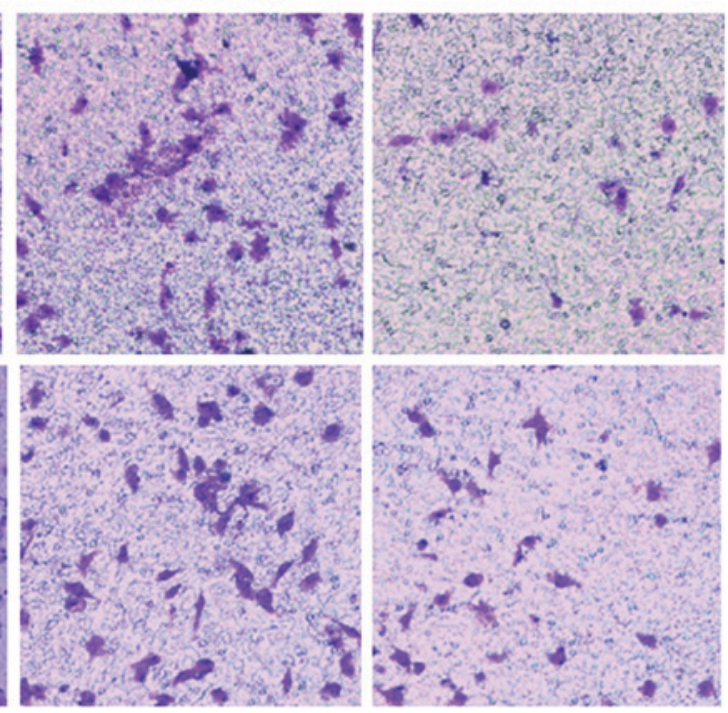

C

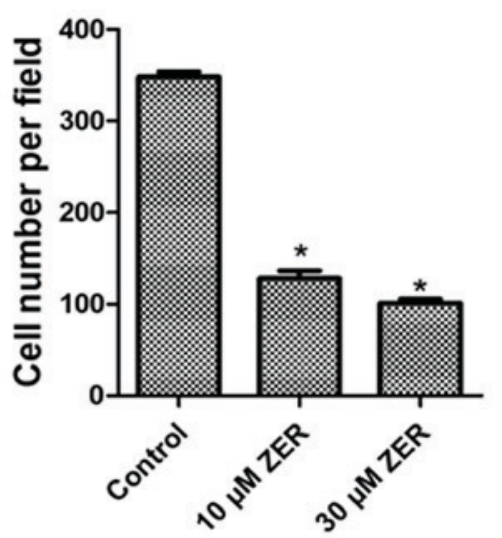

Figure 3. Zerumbone inhibits migration and invasion in HepG2 cells. (A) Cellular migration or invasion was determined using transwell assays. The migratory and invasive abilities of $\mathrm{HepG} 2$ cells were reduced following treatment with zerumbone in a concentration-dependent manner, compared with the control group $(\mathrm{P}<0.05)$. (B) Histogram of the number of migrated cells following treatment with zerumbone for $48 \mathrm{~h}$. (C) Histogram of the number of invaded cells following treatment with zerumbone for $48 \mathrm{~h}$. "P $<0.05$ vs. control.
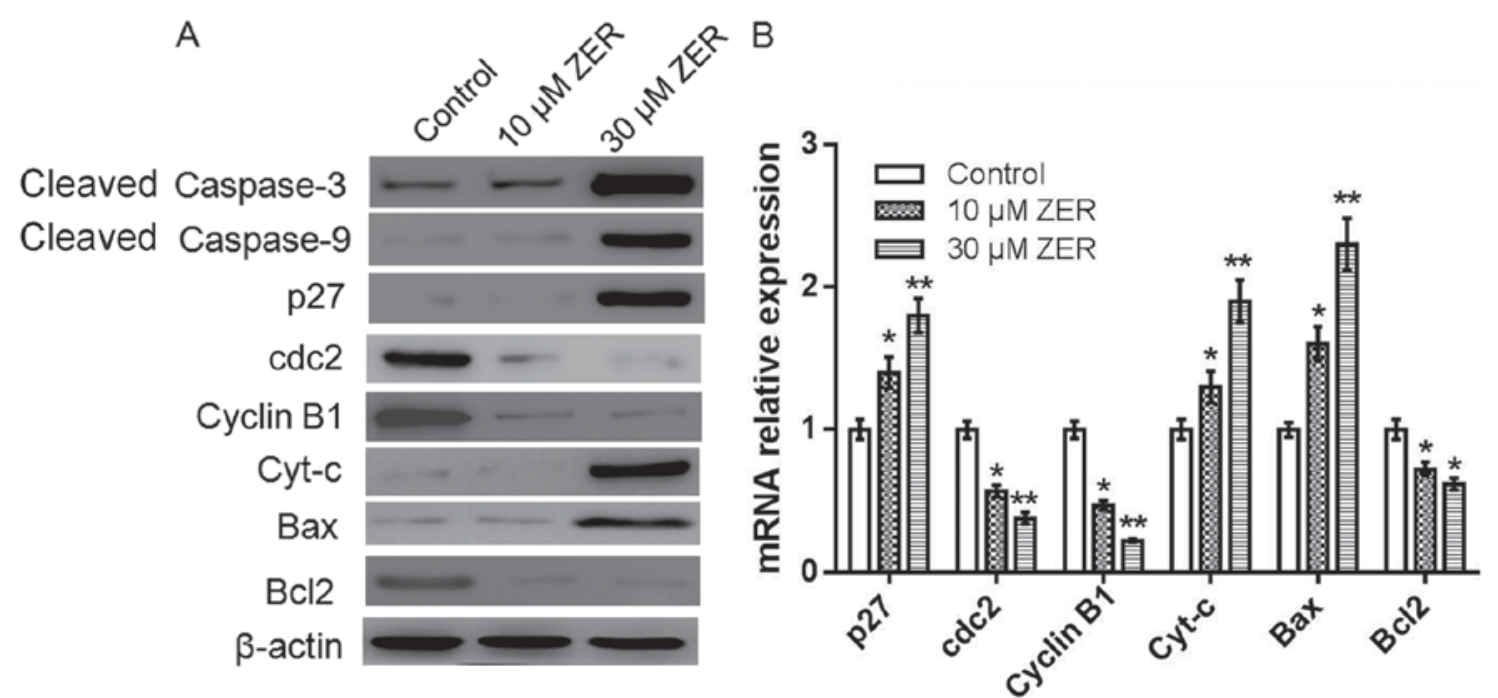

Figure 4. Zerumbone induces activation of caspase-3 and-9 and other apoptosis-associated genes in HepG2 cells. (A) The protein expression of cleaved caspase-3, cleaved caspase-9, p27, CDC2, cyclinB, cytochrome c, Bax and Bcl-2 were measured by western blot analysis. $\beta$-actin was used as an internal control. (B) The mRNA expression of Cyt-c, Bax and Bcl-2 were measured by reverse transcription-quantitative polymerase chain reaction analysis. All experiments were performed in triplicate. ${ }^{*} \mathrm{P}<0.05,{ }^{* *} \mathrm{P}<0.01$ vs. control. p27, cyclin-dependent kinase inhibitor 1B; CDC2, cyclin-dependent kinase 1; Cyt-c, cytochrome c; Bcl-2, B-cell lymphoma-2; Bax, Bcl-2-associated X; ZER, zerumbone. 

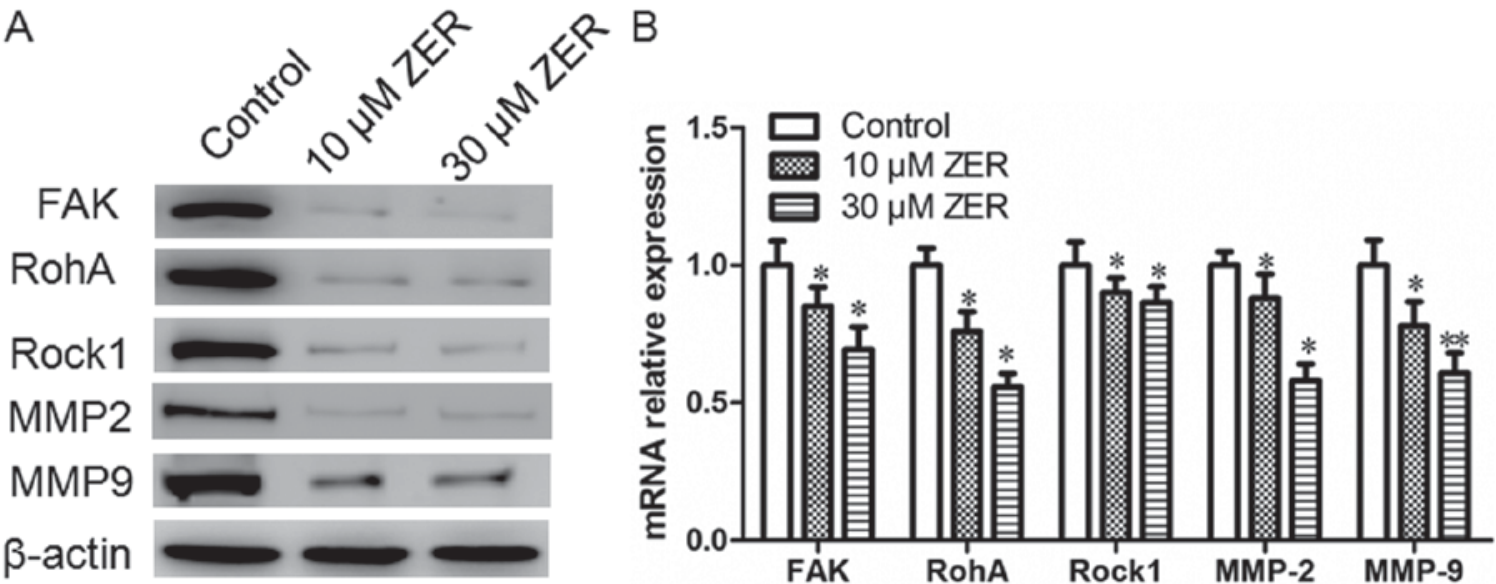

Figure 5. Zerumbone downregulates the expression levels of FAK, RhoA, ROCK-1, MMP2 and MMP9 in HepG2 cells. (A) The protein expression of FAK, RhoA, ROCK-1, MMP2 and MMP9 were measured by western blot analysis. $\beta$-actin was used as an internal control. (B) The mRNA expression of FAK, RhoA, ROCK-1, MMP2 and MMP9 were measured by reverse transcription-quantitative polymerase chain reaction analysis. All experiments were performed in triplicate, ${ }^{, P}<0.05,{ }^{* *} \mathrm{P}<0.01$ vs. control. FAK, focal adhesion kinase; RhoA, Ras homolog gene family, member A; ROCK-1, Rho-associated protein kinase-1; MMP2, matrix metalloproteinase-2; ZER, zerumbone.

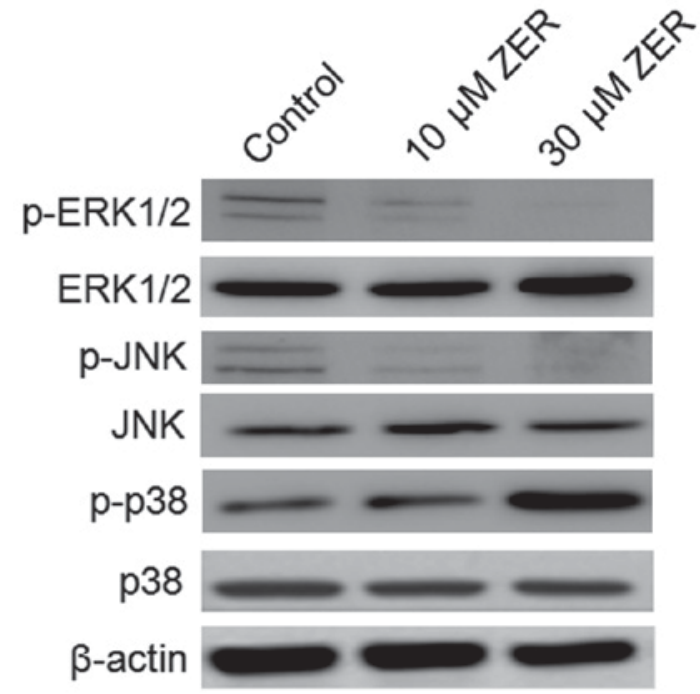

Figure 6. Zerumbone regulated MAPK and ERK phosphorylation in HepG2 cells. HepG2 cells were incubated with zerumbone for $48 \mathrm{~h}$, and the phosphorylated ERK1/2, JNK and p38 was detected by western blot assay. MAPK, mitogen-activated protein kinase $1 \mathrm{ERK}$, extracellular signal-regulated kinase; JNK, c-Jun N-terminal kinase; ZER, zerumbone.

treatment was observed on the regulation of JNK. These findings indicate that inactivation of ERK and activation of p38 MAPK may serve a crucial upstream role in mediating zerumbone activity and inhibiting metastasis in HepG2 cells.

\section{Discussion}

Hepatoma has a high incidence in China and the prevalence was $30-40 \%$ at 5 years postoperatively globally (15). It is difficult to detect during its early stages owing to a lack of symptoms (16). Prognosis for patients with hepatoma is poor owing to the high rate of recurrence and metastasis. Thus, it is essential to investigate the molecular mechanisms driving recurrence and metastasis for improving patient prognosis, and to develop a novel strategy for treating patients with hepatoma by inhibiting specific targets. The present study evaluated the ability of zerumbone to suppress the growth of hepatoma cancer cells in vitro. Zerumbone could significantly inhibit adhesion, migration and invasion in HepG2 cells, and could induce apoptosis. Western blot analysis revealed that zerumbone could induce activation of caspase- 3 , caspase- 9 and and Bax, decrease the expression level of $\mathrm{Bcl}-2$, suppress the expression of MMP-2 and MMP-9, the activity of JNK and ERK was increased, and increase the expression of p-p38 MAPK. These results indicated that zerumbone could inhibit hepatoma growth and metastasis via the MAPK-ERK signal pathway.

To elucidate the mechanism of zerumbone action in hepatoma cells, the expression of cell cycle-and apoptosis-associated genes was examined in HepG2 cells. The release of cytochrome c from the mitochondria into cytoplasm has been considered to be an important event in the apoptotic process mediated by mitochondria (17). In the present study, the data revealed that zerumbone could cause the release of cytochrome c. Induction of apoptosis was associated with the regulation of anti-and pro-apoptotic proteins. Bcl-2 (a notable apoptosis-inhibiting protein) and Bax (an apoptosis-promoting protein) serve key roles in the apoptotic process (18). Induction of Bax expression results in a downstream program of mitochondrial dysfunction, leading to caspase- 9 activation and the subsequent activation of caspase-3 (19). The present study revealed that zerumbone treatment of HepG 2 cells resulted in a dose-dependent decrease in Bcl-2 levels and an increase in Bax. Caspase-3 and-9 were also activated in a dose-dependent manner following zerumbone treatment. These results demonstrated that zerumbone induced mitochondrial activation-mediated cell apoptosis and HepG2 cell death.

The development and progression of tumor metastasis involves a complex multistep process, including cancer cell adhesion, migration and invasion (20). A previous study demonstrated that changes in cytogenetic characteristics are responsible for the invasion and metastasis of cancer cells (21). RhoA, ROCK-1, FAK, MMP-2 and MMP-9 are classical metastasis-associated genes; the effect of zerumbone on 
these molecules was assessed to investigate the underlying mechanism of zerumbone in vitro (22). RhoA, is a small GTPase protein belonging to the Rho family; it is primarily associated with cytoskeletal regulation, mediating actin stress fiber formation and actomyosin contractility (23). ROCK-1 is a serine/threonine kinase that belongs to the Rho family and acts to indirectly diminish the activity of upstream RhoA by stimulating Rac1 activity (24). FAK is involved in cellular adhesion and mediates a key notable early step in cell migration (25). MMP-2 and MMP-9 serve a pivotal role in mediating the malignant behavior of cancer cells, including invasion and metastasis, by degrading the extracellular matrix (26). In the present study, zerumbone was demonstrated to decrease the migration and invasion of hepatoma cells. In addition, the mRNA and protein expression of RhoA, ROCK-1, FAK, MMP-2 and MMP-9 was inhibited following zerumbone treatment in HepG2 cells. These results indicated that suppressing tumor metastasis could be achieved through regulating reorganization of the actin cytoskeleton via Rho GTPase signaling pathways.

The MAPK pathways serve a notable role in mediating the survival of mammalian cells and tumor metastasis (27). The MAPK family includes JNK, p38 MAPK and ERK. p38 MAPK phosphorylation has been implicated to serve a notable role in cell apoptosis, and activation of p38 MAPK decreases ERK1/2 activity (28). The results of the present study demonstrated that the phosphorylation of P38 MAPK was upregulated and phosphorylation of ERK1/2 was downregulated following zerumbone treatment of HepG2 cells but did not exhibit a significant influence on total JNK protein expression. These results indicated that regulation of ERK1/2 and p38 MAPK serves a critical role in restraining cancer cells from invasion and metastasis and inducing apoptosis and cell cycle arrest in response to zerumbone treatment in HepG2 cells.

In conclusion, considering the results of the present study, we hypothesize that zerumbone effectively inhibits the proliferation, and invasion and migration of hepatoma cells in vitro. We hypothesize that the inactivation of ERK1/2 and activation of $\mathrm{p} 38$ MAPK are important initiating signals of the mitochondrial-mediated apoptosis induced and invasion and metastasis restrained by zerumbone. These results indicated that zerumbone might be a potential anticancer agent for the treatment of hepatoma. However, the present study preliminarily investigated several molecules involved in the MAPK pathways, and rescue experiments would be required to confirm the findings and further demonstrate how zerumbone regulates hepatoma invasiveness.

\section{Acknowledgements}

Not applicable.

\section{Funding}

No funding was received.

\section{Availability of data and materials}

All data generated or analyzed during this study are included in this published article.

\section{Authors' contributions}

TL designed the study, WZ performed the experiment and $\mathrm{XH}$ performed the analysis and wrote the paper.

\section{Ethics approval and consent to participate}

Not applicable.

\section{Consent for publication}

Not applicable.

\section{Competing interests}

The authors declare that they have no competing interests.

\section{References}

1. Gong Q, Qin Z and Hou F: Improved treatment of early small hepatocellular carcinoma using sorafenib in combination with radiofrequency ablation. Oncol Lett 14: 7045-7048, 2017.

2. Qiu X, Huang Y, Zhou Y and Zheng F: Aberrant methylation of TRIM58 in hepatocellular carcinoma and its potential clinical implication. Oncol Rep 36: 811-818, 2016.

3. Huang Y, Chen SW, Fan CC, Ting LL, Kuo CC and Chiou JF: Clinical parameters for predicting radiation-induced liver disease after intrahepatic reirradiation for hepatocellular carcinoma. Radiat Oncol 11: 89, 2016.

4. Xiao Y, Tian Q, He J, Huang M, Yang C and Gong L: MiR-503 inhibits hepatocellular carcinoma cell growth via inhibition of insulin-like growth factor 1 receptor. Onco Targets Ther 9: 3535-3544, 2016.

5. Yoo T, Lee KW, Yi NJ, Choi YR, Kim H, Suh SW, Jeong JH, Lee JM and Suh KS: Peri-transplant change in AFP level: A useful predictor of hepatocellular carcinoma recurrence following liver transplantation. J Korean Med Sci 31: 1049-1054, 2016.

6. Sun J, Liu BR, Hu WJ, Yu LX and Qian XP: In vitro anticancer activity of aqueous extracts and ethanol extracts of fifteen traditional Chinese medicines on human digestive tumor cell lines. Phytother Res 21: 1102-1104, 2007.

7. Hsiao WL and Liu L: The role of traditional Chinese herbal medicines in cancer therapy-from TCM theory to mechanistic insights. Planta Med 76: 1118-1131, 2010.

8. Sun YW, Qiu HC, Ou MC, Chen RL and Liang G: Saponins isolated from Schizocapsa plantaginea inhibit human hepatocellular carcinoma cell growth in vivo and in vitro via mitogen-activated protein kinase signaling. Chin J Nat Med 16: 29-40, 2018.

9. Pan JX, Chen G, Li JJ, Zhu QD, Li JJ, Chen ZJ, Yu ZP and Ye LY: Isocorydine suppresses doxorubicin-induced epithelial-mesenchymal transition via inhibition of ERK signaling pathways in hepatocellular carcinoma. Am J Cancer Res 8: 154-164, 2018.

10. Kitayama T: Attractive reactivity of a natural product, zerumbone. Biosci Biotechnol Biochem 75: 199-207, 2011.

11. Rahman HS, Rasedee A, Yeap SK, Othman HH, Chartrand MS, Namvar F, Abdul AB and How CW: Biomedical properties of a natural dietary plant metabolite, zerumbone, in cancer therapy and chemoprevention trials. Biomed Res Int 2014: 920742, 2014.

12. Kirana C, McIntosh GH, Record IR and Jones GP: Antitumor activity of extract of Zingiber aromaticum and its bioactive sesquiterpenoid zerumbone. Nutr Cancer 45: 218-225, 2003.

13. Livak KJ and Schmittgen TD: Analysis of relative gene expression data using real-time quantitative PCR and the 2(-Delta Delta C(T)) method. Methods 25: 402-408, 2001.

14. Sobenin IA, Bobryshev YV, Korobov GA, Borodachev EN, Postnov AY and Orekhov AN: Quantitative analysis of the expression of caspase 3 and caspase 9 in different types of atherosclerotic lesions in the human aorta. Exp Mol Pathol 99: 1-6, 2015.

15. Chen L, Liu H, Liu J, Zhu Y, Xu L, He H, Zhang H, Wang S, Wu Q, Liu W, et al: Klotho endows hepatoma cells with resistance to anoikis via VEGFR2/PAK1 activation in hepatocellular carcinoma. PLoS One 8: e58413, 2013. 
16. Poortahmasebi V, Poorebrahim M, Najafi S, Jazayeri SM, Alavian SM, Arab SS, Ghavami S, Alavian SE, Rezaei Moghadam A and Amiri M: How hepatitis $\mathrm{C}$ virus leads to hepatocellular carcinoma: A network-based study. Hepat Mon 16: e36005, 2016.

17. Garrido C, Galluzzi L, Brunet M, Puig PE, Didelot C and Kroemer G: Mechanisms of cytochrome c release from mitochondria. Cell Death Differ 13: 1423-1433, 2006.

18. Antonsson B: Mitochondria and the Bcl-2 family proteins in apoptosis signaling pathways. Mol Cell Biochem 256-257: 141-155, 2004

19. Deng Y, Lin Y and Wu X: TRAIL-induced apoptosis requires Bax-dependent mitochondrial release of Smac/DIABLO. Genes Dev 16: 33-45, 2002.

20. Stetler-Stevenson WG, Aznavoorian S and Liotta LA: Tumor cell interactions with the extracellular matrix during invasion and metastasis. Annu Rev Cell Biol 9: 541-573, 1993

21. Chang CT, Weng WH, Chou AS, Chuang CK, Porwit-McDonald A Pang ST, Larsson C and Liao SK: Immunophenotypic and molecular cytogenetic features of the cell line UP-LN1 established from a lymph node metastasis of a poorly-differentiated carcinoma. Anticancer Res 25: 683-691, 2005.

22. Lin HJ, Su CC, Lu HF, Yang JS, Hsu SC, Ip SW, Wu JJ, Li YC, Ho CC, Wu CC and Chung JG: Curcumin blocks migration and invasion of mouse-rat hybrid retina ganglion cells (N18) through the inhibition of MMP-2,-9, FAK, Rho A and Rock-1 gene expression. Oncol Rep 23: 665-670, 2010.

23. Ren XD, Kiosses WB and Schwartz MA: Regulation of the small GTP-binding protein Rho by cell adhesion and the cytoskeleton. EMBO J 18: 578-585, 1999.
24. Shi H, Cheng Y, Ye J, Cai P, Zhang J, Li R, Yang Y, Wang Z, Zhang $\mathrm{H}$, Lin C, et al: bFGF promotes the migration of human dermal fibroblasts under diabetic conditions through reactive oxygen species production via the PI3K/Akt-Rac1-JNK pathways. Int J Biol Sci 11: 845-859, 2015.

25. Zhu Y, Zhang X, Qi L, Cai Y, Yang P, Xuan G and Jiang Y: HULC long noncoding RNA silencing suppresses angiogenesis by regulating ESM-1 via the PI3K/Akt/mTOR signaling pathway in human gliomas. Oncotarget 7: 14429-14440, 2016.

26. Bauvois B: New facets of matrix metalloproteinases MMP-2 and MMP-9 as cell surface transducers: Outside-in signaling and relationship to tumor progression. Biochim Biophys Acta 1825: 29-36, 2012.

27. Wagner EF and Nebreda AR: Signal integration by JNK and p38 MAPK pathways in cancer development. Nat Rev Cancer 9: 537-549, 2009.

28. Shi L, Lin Q, Yang T, Nie Y, Li X, Liu B, Shen J, Liang Y, Tang Y and Luo F: Oral administration of Lentinus edodes $\beta$-glucans ameliorates DSS-induced ulcerative colitis in mice via MAPK-Elk-1 and MAPK-PPAR $\gamma$ pathways. Food Funct 7: 4614-4627, 2016.

(c) (i) () This work is licensed under a Creative Commons

c.) Attribution-NonCommercial-NoDerivatives 4.0 International (CC BY-NC-ND 4.0) License. 